(C) EDP Sciences, 2009

DOI: $10.1051 /$ orvie/2009009

\title{
Complexity, emergence and the origins of life
}

\author{
J. Ricard
}

\begin{abstract}
Living organisms, as we know them today, are extremely complex systems and it is highly probable that the same situation prevailed for the first living systems that have appeared on earth. Life is no doubt associated with the emergence of global properties different form those of any of the components of a living system. In order to understand the role played by complexity and emergence in the appearance of life it is no doubt essential to define unambiguously what the term life means. Such a definition, however, is probably impossible to offer and the only sensible approach to our problem is to define what living organisms are or, put in other words, define the main features of living systems.
\end{abstract}

\section{MAIN FEATURES OF LIVING SYSTEMS}

Living organisms are systems that are able to reproduce and hence to give birth to systems similar to them.

These systems possess some kind of specific identity that defines their own organization and functional properties. These functional properties are global features of the system distinct from the individual properties of the component sub-systems. Put in other words, this means that the system can be considered emergent, or complex.

Living systems possess a history viz. they can distinguish, in a sequence of two events, the one that is occurring first. Hence the system can perceive a time arrow and react accordingly.

Living systems are able to evolve under the action of random perturbations.

It is highly probable that these properties are features of both primordial and present living systems. Indeed the simplest chemical system possessing all these features would be a likely candidate for a model of a prebiotic system.

\section{PREBIOTIC SYSTEMS SHOULD BE ABLE TO REPRODUCE}

The question of replication of prebiotic systems is often associated with the idea that nucleic acids can replicate whereas proteins cannot. This idea has led to the proposal of a RNA world and to the view that the first prebiotic systems were basically made up of RNAs (Woese, 1965, 1967; Crick, 1968; Orgel, 1968; Zaug, 1986; Maynard Smith, 1995). In fact, this view could be biased by what we know to occur today. The problem of anteriority of RNAs relative to proteins displays today an obvious circularity as proteins cannot exist without nucleic acids but nucleic acids need enzymes to be synthesized. Hence it is evident that the situation that prevailed during the sequence of events that led to the appearance of life must have been different from those that take place today. In order to avoid this circularity there are two possibilities. The first one is to assume, as already mentioned, that the first living organisms were basically made up of RNAs whereas the second one is to postulate they contained only polypeptides, or proteins. The

Article available at http://www.origins-and-evolution.org or http://dx.doi.org/10.1051/orvie/2009009 
two theories display difficulties. The first one implies that these primordial RNAs should be able both to catalyse chemical reactions, as present day enzymes do, and replicate in the absence of any enzyme. The second one postulates that these primordial proteins catalyse chemical reactions that could spontaneously be duplicated.

When the first theory was experimentally tested, it was shown that some RNAs, called ribozymes (Zaug, 1986), possess the ability to catalyse chemical reactions, although at a slow rate compared to present day enzymes, but were nearly unable to replicate in the absence of any enzyme. This observation does not invalidate the idea of a primordial RNA world for it does not rule out the possibility that some RNAs, which have not been studied so far, possess the ability to replicate spontaneously at a reasonable rate. Nevertheless it is difficult to consider that the available experimental results obtained thus far strongly support the idea of a primordial RNA world.

If we try to consider, in a critical manner, the second hypothesis, it is impossible to assume that the process of "reproduction" of a chemical reaction is due to the replication of the protein catalyst, but it is feasible to postulate it could be due to an activation of protein activity (Desnuelle, 1972). The basic idea put forward by Kauffman (1993, 1995 ) is that these primordial chemical networks should have contained one, or several, autocatalytic reaction(s). Let us consider, for instance, an autocatalytic process such as $A+B \rightarrow X$ in which the product $X$ accelerates its own formation. If this process is included in a sequence of chemical reactions, and if the substrates $A$ and $B$ are continuously renewed, then the whole process will tend to accelerate. From a functional point of view, the present situation is then equivalent to the process of replication. The proposed model seems, at first sight, hypothetical. In fact it is probably not for we know different types of enzyme reactions that precisely display such autocatalysis. Thus, for instance, pepsinogen can be cleaved in vivo into pepsin plus a polypeptide chain. This reaction spontaneously takes place in mammals and is retro-activated by pepsin (Desnuelle, 1972). It then appears that pepsin catalyses its own synthesis. Moreover it seems sensible to assume that reactions that occur in vivo today could have also taken place in prebiotic systems. If such a process had existed it would have resulted in some kind of "replication" of chemical networks. One could have imagined that so-called "biological" properties appeared in a progressive manner as the size of the network increased. According to Kauffman (1993, 1995), this does not seem to be the case. As a matter of fact, the "biological properties" appear at once when a certain size, or complexity, has reached a certain level. Kauffman compares the sudden appearance of "biological properties" to a phase transition, some kind of percolation process (Kauffman, 1993, 1995). This point is illustrated by the following "toy experiment". Let us assume we have on a table a number of buttons as well as pieces of thread. One aims at connecting two buttons by a piece of thread and put the pair down on the same table. If this operation is randomly repeated many times, one should expect on an intuitive basis that, at the beginning of the connecting process, one connects in fact buttons that have not been previously connected. But if we carry on connecting buttons the probability of connecting previously connected buttons increases. On a purely intuitive basis one would expect that the size of the cluster of connected buttons progressively increases as the ratio of the pieces of thread (Th) over the number of buttons (B) increases. This is not what is observed. The size of a cluster of buttons sharply increases as a function of the ratio Th/B. After reaching the 0.5 value it does not vary anymore. A giant cluster is thus formed for 
a definite value of the ratio $\mathrm{Th} / \mathrm{B}$. This process, which is somewhat similar to a phase transition, illustrates, according to Kauffman, the idea that "biological" properties should have appeared when a definite size and complexity of the system has occurred.

\section{PREBIOTIC SYSTEMS POSSESS AN IDENTITY AND CAN DISPLAY EMERGENT PROPERTIES}

We come now to the point of a possible identity of complex chemical networks. Living systems, as they occur today, have an identity which is directly related to the sequence of base pairs in their DNA. Molecular biologists have popularized the view that some elements of this sequence are used as a message that is first coded then transferred in a communication channel, and finally expressed as protein sequences. It is evident however that a chemical network has little to do with the communication of a message in such a way one may wonder whether the concept of information may be related to that of organization. However, the initial definition of the term information, which dates back to Aristotle and scholastic philosophers, is the ability of a system to reach a certain form. According to Aristotle and the scholastic philosophers, the form is the ontological principle that gives the matter the ability to reach a certain organization associated with a given global function. In this perspective, Aristotelian information of a network should be related to the relative importance played by the various nodes in reaching the form, viz. the global organization, of this network. The form is indeed related to the robustness of the network and to the importance played by the various nodes in maintaining its global stability. Hence a difficult question is how to define the importance of the nodes in the expression of the form. If a specific node of a network possesses a high probability of occurrence, slight random perturbations of the system will not result in its fragmentation at the level of this node. Hence one can conclude that such a node has no importance in maintaining the global structure of the system. Conversely, a node of low probability of occurrence is critical for avoiding the breaking up of the network. In this context, information of a node, or of a state, of a system is the relative importance of this node, or of this state, in the evolution of the overall system towards its form. This definition of information is apparently the first one that has been proposed and can still be found in respectable dictionaries such as the Littré's "Dictionnaire de la Langue Française". It is intrinsically different from that used in Shannon's communication theory (Shannon, 1948, 1949).

Let us consider, for instance, a protein edifice that can bind $n$ molecules of ligand $x$ and $n$ molecules of ligand $y$ (Ricard 2006a and b). Then a square lattice $\Omega_{N}$ defined as

$$
\Omega_{N}=\left\{p\left(N_{\kappa, \lambda}\right) ; \kappa, \lambda \in Z^{+}, \kappa, \lambda \leq n\right\}
$$

is formed. Here $p\left(N_{\kappa, \lambda}\right)$ is the probability of occurrence of a node that has bound $\kappa$ molecules of $x$ and $\lambda$ molecules of $y$. In the set $\Omega_{N}$ one can distinguish three different subsets. A first subset $\Omega_{0}$ usually consists of the probability of occurrence of one node only, viz.

$$
\Omega_{0}=\left\{p\left(N_{0,0}\right)\right\}
$$


For the protein edifice has bound neither $x$ nor $y$. The second subset, $\Omega_{N_{x}}$, collects nodes that are of necessity associated with $x$ and, possibly, with $y$, viz.

$$
\Omega_{N_{x}}=\left\{p\left(N_{i, \lambda}\right) ; i \in N, \lambda \in Z^{+}, i, \lambda \leq n\right\}
$$

Last, the third subset, $\Omega_{N_{y}}$, gets together the probabilities of occurrence of nodes whose protein states are all associated with $y$, and possibly with $x$. Hence one has

$$
\Omega_{N_{y}}=\left\{p\left(N_{\kappa, j}\right) ; \kappa \in Z^{+}, j \in N, \kappa, j \leq n\right\}
$$

The intersection of the two subsets $\Omega_{N_{x}}$ and $\Omega_{N_{y}}$ is then

$$
\Omega_{N_{x y}}=\Omega_{N_{x}} \cap \Omega_{N_{y}}=\left\{p\left(N_{i, j}\right) ; i, j \in N, i, j \leq n\right\}
$$

And its nodes all bear $x$ and $y$. As the network is supposed to be a lattice, there are several nodes, $N_{i, j}$, associated with the same number, $i$, of $x$ molecules but a variable number, $\lambda$, of $y$ molecules. One can then define the probability of a state, $p\left(x_{i}\right)$, as

$$
p\left(x_{i}\right)=\sum_{\lambda} p\left(N_{i, \lambda}\right) \quad\left(i \in N ; \lambda \in Z^{+}\right)
$$

This probability is the probability that the proteins have bound $i$ molecules of $x$ whether or not they have also bound molecules of $y$. Hence this probability is the probability of a state made up of a number of nodes that all bear $i$ molecules of ligand $x$ but possibly also molecules $y$. As the value of $p\left(x_{i}\right)$ does not depend upon the different types of interactions that may exist between the two types of ligands, one can conclude that the value of $p\left(x_{i}\right)$ does not reflect any particular type of organization, or of interaction, of ligands $x$ and $y$.

Similarly, one can define the probability

$$
p\left(y_{j}\right)=\sum_{\kappa} p\left(N_{\kappa, j}\right) \quad\left(j \in N ; \kappa \in Z^{+}\right)
$$

which is the probability that the proteins have bound $j$ molecules of $y$ whether or not they have bound $x$. Exactly as previously mentioned for $p\left(x_{i}\right)$, the value of $p\left(y_{j}\right)$ does not reflect any particular type of organization of ligands $x$ and $y$. From relationships (6) and (7) one can define two sets $\Omega_{X}$ and $\Omega_{Y}$, viz.

$$
\begin{aligned}
& \Omega_{X}=\left\{p\left(x_{i}\right) ; i \in N\right\} \\
& \Omega_{Y}=\left\{p\left(y_{j}\right) ; j \in N\right\}
\end{aligned}
$$

The corresponding states $x_{i}$ and $y_{j}$ allow one to define two sets $X$ and $Y$ whose Cartesian product is $X Y$. The probability space of this system is

$$
\Omega_{X Y}=\left\{p\left(x_{i}, y_{j}\right) ; i, j \in N\right\}
$$


We have seen that the smaller the probability of occurrence of a state and the larger its importance. This idea can be expressed by the following relationships

$$
\begin{aligned}
h\left(x_{i}\right) & =f\left\{\frac{1}{p\left(x_{i}\right)}\right\} \\
h\left(y_{j}\right) & =f\left\{\frac{1}{p\left(y_{j}\right)}\right\} \\
h\left(x_{i}, y_{j}\right) & =f\left\{\frac{1}{p\left(x_{i}, y_{j}\right)}\right\}
\end{aligned}
$$

where $h\left(x_{i}\right), h\left(y_{j}\right)$ and $h\left(x_{i}, y_{j}\right)$ are the importance, or the Aristotelian information, of the corresponding states (or nodes) in defining the form of the overall system. So far in our reasoning, $f$ is an unknown function. Moreover it is evident that when two ligands are involved in the expression of a state each of them should play a certain part in the information of this state. This means that

$$
h\left(x_{i}, y_{j}\right)=h\left(x_{i}\right)+h\left(y_{j} \mid x_{i}\right)=h\left(y_{j}\right)+h\left(x_{i} \mid y_{j}\right)
$$

The simplest way to accommodate conditions (10) and (11) is to assume that the function $f$ of expressions (10) is a logarithmic one. It follows that

$$
\begin{aligned}
h\left(x_{i}\right) & =-\log p\left(x_{i}\right) \\
h\left(y_{j}\right) & =-\log p\left(y_{j}\right) \\
h\left(x_{i}, y_{j}\right) & =-\log p\left(x_{i}, y_{j}\right)=-\log p\left(x_{i}\right)-\log p\left(y_{j} \mid x_{i}\right)
\end{aligned}
$$

Moreover, it is clear from relations (11) that the interactions between $x_{i}$ and $y_{j}$ should affect the importance, viz. the Aristotelian information, of nodes bearing both $x_{i}$ and $y_{j}$. In order to evaluate the effect of the interaction between $x_{i}$ and $y_{j}$ on the information of the corresponding node, one can define another variable, $i\left(x_{i}: y_{j}\right)$ as,

$$
i\left(x_{i}: y_{j}\right)=h\left(x_{i}\right)+h\left(y_{j}\right)-h\left(x_{i}, y_{j}\right)
$$

This is the variation of Aristotelian information, positive or negative, associated with the formation of a node involving two ligands $x_{i}$ and $y_{j}$. Taking advantage of equations (11), expression (13) can be rewritten as

$$
i\left(x_{i}: y_{j}\right)=h\left(x_{i}\right)-h\left(x_{i} \mid y_{j}\right)=h\left(y_{j}\right)-h\left(y_{j} \mid x_{i}\right)
$$

Whereas $h\left(x_{i}\right)$ is the information of a state that has bound $i$ molecules of $x$, whether or not it has already bound molecules of $y, h\left(x_{i} \mid y_{j}\right)$ represents the information of a node that has bound $i$ molecules of $x$ given it has already bound $j$ molecules of $y$. If $h(x \mid y)<h(x)$, the interaction of $x$ and $y$, namely their mutual organization, results in the consumption of information in the global system. Conversely, if $h(x \mid y)$ is larger than $h(x)$ the interaction of the two ligands brings about the emergence of an information in the same global system. 
The above reasoning can be extended to a population of nodes of a network. One can define functions

$$
\begin{aligned}
& H(X)_{N}=\sum_{i} \sum_{j} p\left(x_{i}, y_{j}\right) \cdot h\left(x_{i}\right) \\
& H(Y)_{N}=\sum_{i} \sum_{j} p\left(x_{i}, y_{j}\right) \cdot h\left(y_{i}\right)
\end{aligned}
$$

that express the information of sets of $x$ and $y$ states, respectively. By convention, these relationships are expressed per node that has bound both ligands $x$ and $y$. Care must be taken that these expressions are not entropies for one has

$$
\begin{aligned}
& \sum_{i} p\left(x_{i}, y_{j}\right) \leq p\left(x_{i}\right) \\
& \sum_{j} p\left(x_{i}, y_{j}\right) \leq p\left(y_{j}\right)
\end{aligned}
$$

As a matter of fact, $p\left(x_{i}\right)$ and $p\left(y_{j}\right)$ are probabilities of states whereas $p\left(x_{i}, y_{j}\right)$ is the probability of a node.

In the same line, one can define conditional information of set of nodes

$$
\begin{aligned}
& H(X \mid Y)_{N}=\sum_{i} \sum_{j} p\left(x_{i}, y_{j}\right) \cdot h\left(x_{i} \mid y_{j}\right) \\
& H(Y \mid X)_{N}=\sum_{i} \sum_{j} p\left(x_{i}, y_{j}\right) \cdot h\left(y_{j} \mid x_{i}\right)
\end{aligned}
$$

As previously, these functions have values conventionally expressed per node associating the two ligands $x$ and $y$. By extension of a previous reasoning (equation 14) to a set of nodes, one can define a so-called mutual information of integration, $I(X: Y)_{N}$, as

$$
I(X: Y)_{N}=H(X)_{N}-H(X \mid Y)_{N}=H(Y)_{N}-H(Y \mid X)_{N}
$$

If the function $I(X: Y)_{N}$ has positive values, the organization of the system requires the consumption of information. If, conversely, $I(X: Y)_{N}<0$, the organization of the system spontaneously generates information. In the first case the system is defined as integrated whereas it is considered emergent in the second case. Last, if one has $I(X: Y)_{N}=0$, the $x$ and $y$ do not interact and the network cannot be considered a system. In the scope of emergence of Aristotelian information one should expect that this process be associated with the appearance of a novel function. We shall discuss this point later.

\section{PREBIOTIC SYSTEMS SHOULD POSSESS A HISTORY AND SHOULD BE ABLE TO SENSE SIGNALS}

Exactly as for living organisms, prebiotic systems should be able to possess a history, viz. they should be able to distinguish, in a sequence of events, the one that is occurring first. As we shall see later, such an ability is often related to the capacity of the system to sense signals and, for that reason, it is of interest not to distinguish between these two properties. It has been outlined that the fundamental equations of physics, viz. those that describe the time evolution of elementary processes, remain unchanged if the variable $t$ is replaced 

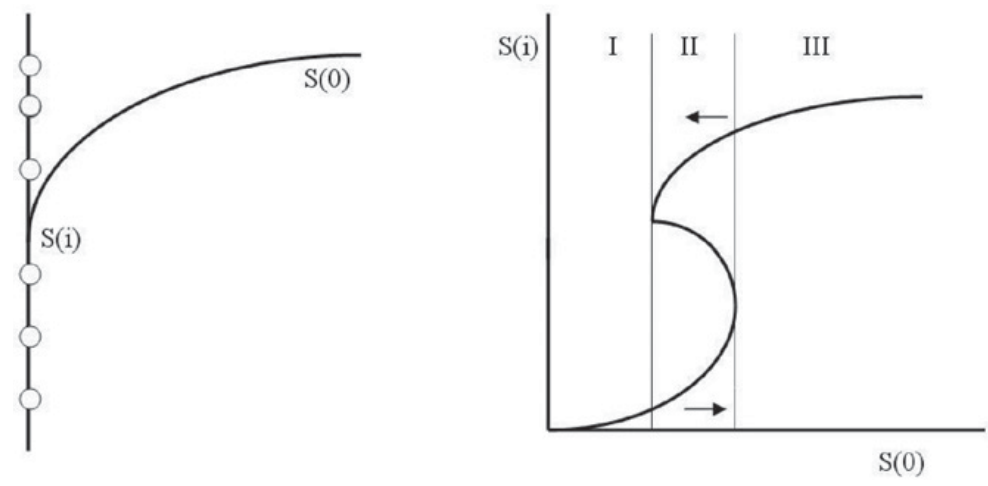

Figure 1. Left: The enzyme molecules are bound to an impermeable surface. A concentration gradient is formed in the vicinity of the surface. Right: The system can display multiple steady states. In region II, the system is able to sense the direction of a concentration change (orientation of the arrow).

by $-t$. On the contrary, living systems should be considered in the context of a time arrow. One can be tempted to think that prebiotic systems should be looked on the same way. Let us consider for instance the apparently simple system made up of an enzyme reaction coupled to the diffusion of its substrate (Figure 1). In this system the enzyme molecules are bound to an impermeable membrane and the substrate of the enzyme slowly diffuses towards the bound enzyme. If the overall system is under steady state conditions and if the rate equation is nonlinear, one can demonstrate that the coupled process can display several steady states, three for instance. Such a situation gives the system the ability to sense whether the external concentration, far from the surface, increases or decreases. In region II (Figure 1), for instance, the system reacts differently depending on the bulk concentration of the substrate is increased or decreased. Conversely, in regions I and III, it cannot detect the direction of a concentration change. This means that in region II the system is able to perceive a time arrow whereas it cannot in the two regions I and III.

Perceiving a time evolution, or a time arrow, is somewhat similar to the perception of physical and chemical signals for the system should be able to sense both the direction and the intensity of a physical, or a chemical signal. This is equivalent to the recognition of the time arrow. It is remarkable that apparently simple systems that display multiple steady states can possess the ability to sense whether a signal has an increasing, or decreasing, intensity. Hence the behaviour of such systems is clearly taking place in the context of a time arrow, which is no doubt an important feature of living systems.

\section{PREBIOTIC SYSTEMS SHOULD EVOLVE}

It appears very likely that prebiotic systems must have been subjected to some kind of primitive evolution. If a network made up of many chemical reactions surrounded by a membrane can be considered a typical prebiotic system, such a system should be 


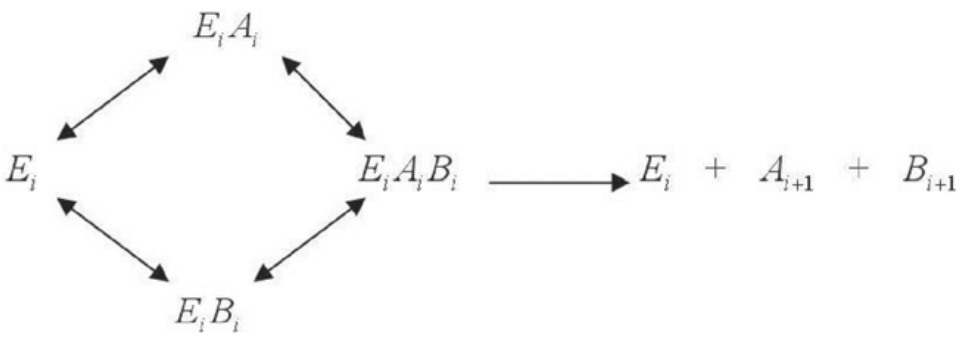

Figure 2. A likely enzyme reaction involving two substrates.

subjected to spontaneous dismantling. Hence a first, but essential, step of the evolution of such prebiotic systems should be a global increase of their robustness. As a matter of fact, a network of catalysed (for instance enzyme-catalysed) chemical reactions can be considered a meta-network for the processes involved in individual enzyme reactions are usually fast relative to the processes that connect these reactions. Owing to this time hierarchy, any chemical reaction can be considered a node of the meta-network whereas the connections of these reactions are the edges of the meta-network. The strength of the edge connecting two nodes $N_{i}$ and $N_{i+1}$ (two chemical reactions) of the meta-network relies upon the product of the apparent catalytic constant of the reaction (the node $N_{i}$ ) by the joint probability that enzyme $E_{i}$ has bound its two substrates $A_{i}$ and $B_{i}$. Hence robustness of the network between nodes $N_{i}$ and $N_{i+1}$ relies upon the values of the product $k_{i} \cdot p\left(A_{i}, B_{i}\right)$ where $k_{i}$ and $p\left(A_{i}, B_{i}\right)$ are the above mentioned catalytic constant and joint probability, respectively. If one of the values of this product drops below a certain critical limit the meta-network dismantles. Hence it is important to look after the physical conditions that control the values of this product. Moreover the important question is to know how can a biochemical network increase its robustness? Let us apply these general ideas to a simple biochemical network.

The simplest biochemical network one can think of is an enzyme reaction. When an enzyme catalyses a chemical reaction of the type $A_{i}+B_{i} \rightarrow A_{i+1}+B_{i+1}$ a likely succession of events is the binding of the two substrates, $A_{i}$ and $B_{i}$, to the enzyme thus making easier the chemical reaction between them to occur. If, for instance, the enzyme has bound $A_{i}$ first it may bind $B_{i}$ afterwards, and conversely (Figure 2). A ternary complex involving the enzyme and the two substrates is formed and the chemical reaction between $A_{i}$ and $B_{i}$ takes place at the surface of the enzyme molecule. Such a system displays some sort of organization if the binding of $A_{i}$ to the enzyme affects that of $B_{i}$, and conversely. The ability of such a system to display this kind of organization implies the consumption, or alternatively the supply, of information. In such a system the probabilities of occurrence of states $A_{i}, B_{i}$ and $A_{i} B_{i}$ are

$$
\begin{aligned}
p\left(A_{i}\right) & =p\left(E_{i} A_{i}\right)+p\left(E_{i} A_{i} B_{i}\right) \\
p\left(B_{i}\right) & =p\left(E_{i} B_{i}\right)+p\left(E_{i} A_{i} B_{i}\right) \\
p\left(A_{i}, B_{i}\right) & =p\left(E_{i} A_{i} B_{i}\right)
\end{aligned}
$$


If the system is not too far away from thermodynamic equilibrium conditions one finds

$$
\begin{aligned}
p\left(A_{i}\right) & =\frac{K_{A}\left[A_{i}\right]\left(1+K_{B}^{\prime}\left[B_{i}\right]\right)}{1+K_{A}\left[A_{i}\right]+K_{B}\left[B_{i}\right]+K_{A} K_{B}^{\prime}\left[A_{i}\right]\left[B_{i}\right]} \\
p\left(B_{i}\right) & =\frac{K_{B}\left[B_{i}\right]\left(1+K_{A}^{\prime}\left[A_{i}\right]\right)}{1+K_{A}\left[A_{i}\right]+K_{B}\left[B_{i}\right]+K_{A} K_{B}^{\prime}\left[A_{i}\right]\left[B_{i}\right]} \\
p\left(A_{i}, B_{i}\right) & =\frac{K_{A} K_{B}^{\prime}\left[A_{i}\right]\left[B_{i}\right]}{1+K_{A}\left[A_{i}\right]+K_{B}\left[B_{i}\right]+K_{A} K_{B}^{\prime}\left[A_{i}\right]\left[B_{i}\right]}
\end{aligned}
$$

In these expressions the Ks are the binding (equilibrium) constants of the substrates to the enzyme forms. Moreover the equilibrium constants are not independent for thermodynamics requires that

$$
K_{A} K_{B}^{\prime}=K_{A}^{\prime} K_{B}
$$

It is then possible to derive the expression of the corresponding Aristotelian information of states $A_{i}, B_{i}$ and $A_{i} B_{i}$. One has

$$
\begin{aligned}
H\left(A_{i}\right) & =-\log p\left(A_{i}\right) \\
H\left(B_{i}\right) & =-\log p\left(B_{i}\right) \\
H\left(A_{i}, B_{i}\right) & =-\log p\left(A_{i}, B_{i}\right)
\end{aligned}
$$

One can also define conditional informations $H\left(A_{i} \mid B_{i}\right)$ and $H\left(B_{i} \mid A_{i}\right)$ from the corresponding conditional probabilities. One finds

$$
\begin{aligned}
& H\left(A_{i} \mid B_{i}\right)=-\log p\left(A_{i} \mid B_{i}\right)=\frac{-\log K_{A}^{\prime}\left[A_{i}\right]}{1+K_{A}^{\prime}\left[A_{i}\right]} \\
& H\left(B_{i} \mid A_{i}\right)=-\log p\left(B_{i} \mid A_{i}\right)=\frac{-\log K_{B}^{\prime}\left[B_{i}\right]}{1+K_{B}^{\prime}\left[B_{i}\right]}
\end{aligned}
$$

One can derive the expression of the mutual information of integration as

$$
I_{i}\left(A_{i}: B_{i}\right)=\log \frac{p\left(A_{i} \mid B_{i}\right)}{p\left(A_{i}\right)}
$$

and one finds

$$
I_{i}\left(A_{i}: B_{i}\right)=\log \frac{1+K_{A}\left[A_{i}\right]+K_{B}\left[B_{i}\right]+K_{A} K_{B}^{\prime}\left[A_{i}\right]\left[B_{i}\right] K_{B}^{\prime}}{1+K_{A}^{\prime}\left[A_{i}\right]+K_{B}^{\prime}\left[B_{i}\right]+K_{A}^{\prime} K_{B}^{\prime}\left[A_{i}\right]\left[B_{i}\right] K_{B}}
$$

One can see that the sign of this expression depends upon the respective values of $K_{A}$ and $K_{A}^{\prime}$ as well as $K_{B}$ and $K_{B}^{\prime}$. If $K_{A}^{\prime}>K_{A}$, which implies that $K_{B}^{\prime}>K_{B}$, then $I_{i}\left(A_{i}: B_{i}\right)>0$ and the system is called integrated. If conversely $K_{A}^{\prime}<K_{A}$, which means also that $K_{B}^{\prime}<K_{B}$, then $I_{i}\left(A_{i}: B_{i}\right)<0$ and the system is defined as emergent. In the first case the chemical system requires information and in the second case it generates information. Last if $K_{A}^{\prime}=K_{A}$ and $K_{B}^{\prime}=K_{B}$ the reaction neither consumes, nor generates information. In that case the whole process cannot be considered a system.

It is now possible to raise the critical point of the effect of the emergernce of a node of the meta-network upon its stability. We have seen above that the connexion of nodes $N_{i}$ and $N_{i+1}$ in a meta-network is expressed by the product $k_{i} p\left(A_{i}, B_{i}\right)$. Taking advantage of 
the last expression (20), one can see that this product is equal to

$$
k_{i} p\left(A_{i}, B_{i}\right)=\frac{k_{i} K_{A} K_{B}^{\prime}\left[A_{i}\right]\left[B_{i}\right]}{1+K_{A}\left[A_{i}\right]+K_{B}\left[B_{i}\right]+K_{A} K_{B}^{\prime}\left[A_{i}\right]\left[B_{i}\right]}
$$

One can easily conclude that if one raises the energy level of the ternary state $E_{i} A_{i} B_{i}$ in such a way it approaches that of the corresponding transition state, then the value of the corresponding catalytic constant $k_{i}$ increases but that of the product $K_{A} K_{B}^{\prime}$ declines in such a way that the product $k_{i} K_{A} K_{B}^{\prime}$ remains unchanged. As the constants $K_{A}$ and $K_{B}$ do not vary, it follows from this situation that expression (26) increases. Moreover a decline of $K_{A} K_{B}^{\prime}$ (or $K_{A}^{\prime} K_{B}$ ) at constant value of $K_{A}$ (and $K_{B}$ ) means that the relationships $K_{A}>K_{A}^{\prime}$ and $K_{B}>K_{B}^{\prime}$ tend to apply as the energy level of the $E_{i} A_{i} B_{i}$ state approaches that of the corresponding transition state. It follows from this reasoning that the emergence of a meta-network tends to increase its stability. Conversely, the appearance of integration of nodes tends to induce network dismantling.

\section{GENERAL DISCUSSION AND CONCLUSIONS}

Most theories on the origin of life rely on the putative existence of a RNA world in which the same RNA molecules were able both to store information and to catalyse chemical reactions. Some time ago, however, Kauffman $(1993,1995)$ has proposed the view that networks of chemical reactions surrounded by a membrane could be likely candidates for prebiotic systems. In order to possess such an ability, chemical networks should meet, as already outlined, the following requirements: they should be able to reproduce, they should possess an identity as all living systems do, they should be able to display a history and, as a consequence, to sense signals, and, last but not least, they should evolve.

The first requirement, namely the reproduction of the system, does not represent much difficulty if one assumes that a number of chemical reactions that take part in the network are autocatalytic viz. their products stimulate their own formation. There is little risk speculating on this property for we know, in present day world, biochemical reactions that precisely display this property.

The second requirement is more tricky as the identity of living systems relies today upon the base sequence of their DNA and one could indeed wonder about what could be the identity of a chemical, or a biochemical, network that obviously neither contain any DNA nor RNA. Studies on system organization and emergence (Ricard, 2006, 2006, 2008) show that networks possess some kind of Aristotelian information itself expressed by the importance of the various nodes in maintaining the global integrity, the form, of the network. This form, which is required for an optimal collective function of the network, can be considered the identity of this network that can be given a quantitative expression through the function $I(X: Y)_{N}$. As a matter of fact, this function expresses the Aristotelian information of the nodes of the network and how possible interaction of different ligands affects this information.

Living systems possess a history. Thus, for instance, they are able to sense the intensity of a signal but are also able to sense the direction of a change of intensity. Being able to sense whether the same intensity is reached after an increase, or a decrease, of intensity is a property related to the existence of a time arrow. This kind of property can be found and described in many biomimetic systems. It can be easily explained on the basis of 
their nonlinear character (Prigogine, 1968, 1996; Nicolis, 1977). The recognition of a time arrow is probably present in all living organisms and has probably been an essential feature of prebiotic systems.

The last property that prebiotic systems should possess is the ability to evolve. Hence one can raise the question to know what could be understood by evolution of a chemical, or a biochemical, network. As it is evident that the function of a network is, of necessity, a collective property of a set of connected chemical, or biochemical, reactions, one can think of the evolution of such a network as a trend towards an increased robustness. One of the results presented in this paper is that networks of chemical, or biochemical, reactions are in fact meta-networks whose nodes are chemical, or biochemical, reactions. Meta-networks can increase their robustness by increasing the emergent character of their nodes.

One might perhaps be stuck by the speculative character of the above theoretical considerations. This feature, however, is probably a general feature of all the studies devoted to the problem of the origin of life. But these speculations are far from gratuitous for they are based on sound physical foundations which, I believe, is the only critical requirement for any consistent study on the origin of life.

\section{References}

Crick, F.H.C., 1968. J. Mol. Biol. 38, 367-379.

Desnuelle, P., 1972. In The Enzymes. 3rd edn. P. D. Boyer ed. 7, 575-586.

Kauffman, S., 1993. The Origins of Order. Oxford University Press.

Kauffman, S., 1995. At Home in the Universe. Penguin Books.

Maynard Smith, J., Szathmary, E., 1995. The Major Transitions in Evolution. Oxford University Press.

Nicolis, G., Prigogine, I., 1977. Self-organization in Nonequilibrium Systems. Wiley and Sons.

Orgel, L.E., 1968. J. Mol. Biol. 38, 381-393.

Prigogine, I., 1968. Introduction à la Thermodynamique des Processus Irreversibles. Dunod, Jacques Gabay, Paris.

Prigogine, I., 1996. La Fin des Certitudes. Editions Odile Jacob, Paris.

Ricard, J., 2006a. Journ. Non-equil. Thermodyn. 31, 103-152.

Ricard, J., 2006b. Emergent Collective Properties, Networks and Information in Biology. Elsevier, Asterdam, New York.

Ricard, J., 2008. Pourquoi le Tout est plus que la Somme de ses Parties. Hermann, Paris.

Shannon, C.E., 1948. A mathematical theory of communication. Bell System Technical Journal. 27, 379-423.

Shannon, C.E., 1948. A mathematical theory of communication. Bell System Technical Journal. 27-623-656.

Shannon, C.E., 1949. The Mathematical Theory of Communication. University of Illinois Press, Urbana.

Woese, C.K., 1965. Proc. Natl. Acad. Sci. 77, 1083-1086.

Woese, C.K., 1967. The Genetic Code: the Molecular Basis for Genetic Expression. Harper and Row, New York.

Zaug, A.J., Cech, T.R., 1986. Science. 231, 470-475. 